\title{
MONOTONICITY OF COEFFICIENTS OF RECIPROCAL POWER SERIES
}

\begin{abstract}
Given a power series $f(x)=\sum_{n=1}^{\infty} a_{n} x^{n}$ with nonnegative coefficients satisfying $\sum_{n=1}^{\infty} a_{n}=1$ we give sufficient conditions on the sequence $\left(a_{n}\right)$ to guarantee that the coefficients of the Taylor series of $h(x)=1 /(1-f(x))$ form a nonincreasing sequence. This type of result is useful when one wishes to apply Tauberian theorems.
\end{abstract}

\section{Introduction}

The purpose of this article is to investigate the following question. Given a series of nonnegative terms satisfying $\sum_{n=1}^{\infty} a_{n}=1$ let $f(x)=\sum_{n=1}^{\infty} a_{n} x^{n}$ and define

$$
h(x)=\frac{1}{1-f(x)}=\sum_{n=0}^{\infty} p_{n} x^{n} .
$$

The problem is to determine conditions on the given series under which $\left(p_{n}\right)$ is a nonincreasing sequence. We give sufficient conditions for this, which, although not necessary, are easy enough to check, and apply to important examples in which the monotonicity of the coefficients is needed. In particular we show (Corollary 3.4) that if $\left(a_{n+1}\right)^{2} \leq a_{n} a_{n+1}$ for all $n \geq 1$, then $\left(p_{n}\right)$ is a nonincreasing sequence.

What motivated the present investigation and is also the type of application we have in mind, is to help verify that certain requirements are met in order to apply Tauberian theorems. In the study of functions represented by power series, a theorem is called Abelian if properties of the represented functions

Key Words: Monotonicity of Coefficients, Renewal Equation, Tauberian Theorems

Mathematical Reviews subject classification: 30B10, 65Q05, 39B62

Received by the editors May 16, 2000

*This work was done while the author was visiting the University of Delaware. 
are found from properties of the coefficients. A theorem is called Tauberian if properties of the coefficients are found from properties of the function. The following is a very useful theorem of Tauberian type.

Theorem 1.1 ([5], pg. 33). Let $\sum_{n=0}^{\infty} p_{n} x^{n}$ be a power series converging for $|x|<1$. Suppose that the coefficients $p_{n}$ are nonnegative and nonincreasing. Suppose in addition that for some $\beta$, with $0<\beta<1$,

$$
\sum_{n=0}^{\infty} p_{n} x^{n}=\frac{1}{(1-x)^{\beta}}+o\left(\frac{1}{(1-x)^{\beta}}\right) .
$$

Then $\lim _{n \rightarrow \infty} \Gamma(\beta) n^{1-\beta} p_{n}=1$.

This theorem is a consequence of the famous Tauberian theorem of Hardy and Littlewood, which in turn is a converse of Appell's theorem of Abelian type.

Theorem 1.2 (Appell's Theorem, [5], pg. 12). Let $\delta \geq 0$ and $A$ be constants and let $S_{N}=\sum_{n=0}^{N} b_{n} \approx A N^{\delta} / \Gamma(1+\delta)$, as $N \rightarrow \infty$. Then

$$
\lim _{x \rightarrow 1^{-}}(1-x)^{\delta} \sum_{n=0}^{\infty} b_{n} x^{n}=A
$$

Here the symbol $s_{n} \approx t_{n}$ stands for $\lim s_{n} / t_{n}=1$.

We now explain how our theorem can be employed in the application of Tauberian results. A common situation in which Tauberian theorems are used is in the study of renewal type equations. Given sequences $\left(k_{n}\right)$ and $\left(g_{n}\right)$, one would like to be able to say something about the rate of convergence to zero of the sequence $\left(p_{n}\right)$, where $p_{n}$ satisfies

$$
p_{n}=k_{n}+\sum_{j=1}^{n-1} p_{n-j} g_{j}
$$

Consider the power series

$$
h(x)=\sum_{n=1}^{\infty} p_{n} x^{n}, g(x)=\sum_{n=1}^{\infty} g_{n} x^{n}, \text { and } k(x)=\sum_{n=1}^{\infty} k_{n} x^{n} .
$$

From the recursive equation for $p_{n}$ above we get $h(x)=k(x)+g(x) h(x)$, and, therefore, $h(x)=\frac{k(x)}{1-g(x)}$. Suppose that $\sum_{n=1}^{\infty} g_{n}=1$ and that $S_{N}=\sum_{n=1}^{N} k_{n}$ 
behaves as $S_{N} \approx C \cdot N^{\delta}$, when $N \rightarrow \infty$, for some $\delta$ with $0<\delta<1$ and some $C>0$. Setting $\beta=1-\delta$ we get

$$
\frac{h(x)}{(1-x)^{-\beta}}=\frac{k(x)}{(1-x)^{-\beta+1}} \frac{1-x}{1-g(x)} .
$$

By Theorem 1.2 the limit of $k(x) /(1-x)^{-\beta+1}$ as $x \rightarrow 1^{-}$exists.

If $g_{n}$ converges to zero fast enough (for example, $g_{n}=O\left(n^{-(1+b)}\right)$, with $b>1$ ), then $g(x)$ is differentiable at $x=1$ (see [1], Vol. II). This assures the existence of the limit of $(1-x) /(1-g(x))$ as $x \rightarrow 1^{-}$.

The conclusion is that $\lim _{x \rightarrow 1^{-}} \frac{h(x)}{(1-x)^{-\beta}}$ exists. Now in order to use Theorem 1.2 and obtain an estimate of the rate of convergence of $p_{n}$ to 0 , we need $\left(p_{n}\right)$ to be monotone.

When $p_{n}$ is obtained by means of

$$
h(x)=\frac{1}{1-f(x)},
$$

with $f(x)=\sum_{n=1}^{\infty} a_{n} x^{n}$, our theorem can be used to establish monotonicity of $\left(p_{n}\right)$.

Indeed, our result was applied with this purpose by A. Fisher and A. Lopes in [2], to estimate a lower bound for the polynomial decay of correlation for the Gibbs measure of a certain one-dimensional dynamical system with an indifferent fixed point. This system is a piecewise linear version of the Manneville-Pomeau map (see [4]). More precisely, for $T$ a two-to-one map of the interval $[0,1]$ to itself, and $\psi:[0,1] \rightarrow \mathbb{R}$ a non-Hölder potential, the associated Ruelle-Perron-Frobenius operator $\mathcal{L}$, defined by

$$
(\mathcal{L} \varphi)(x)=\sum_{T(y)=x} e^{\psi(y)} \varphi(y),
$$

is known to satisfy

$$
\lim _{n \rightarrow \infty}\left(\mathcal{L}^{n} \varphi\right)(x)=\int \varphi(y) d \mu(y)
$$

for any $x \in[0,1]$ and observable $\varphi:[0,1] \rightarrow \mathbb{R}$, where $\mu$ is the equilibrium measure for the potential $\psi$. In [2], in order to estimate the velocity of decay of the $n$th order correlation

$$
\begin{aligned}
c_{n} & =\int \varphi\left(T^{n}(x)\right) \varphi(x) d \mu(x)-\left(\int \varphi(y) d \mu(y)\right)^{2} \\
& =\int \varphi(x)\left(\mathcal{L}^{n} \varphi\right)(x) d \mu(x)-\int \varphi(x)\left(\int \varphi(y) d \mu(y)\right) d \mu(x) \\
& =\int \varphi(x)\left(\mathcal{L}^{n} \varphi(x)-\int \varphi(y) d \mu(y)\right) d \mu(x),
\end{aligned}
$$


an estimate is needed for the velocity of convergence in $n$ of the term

$$
d_{n}=\left(\mathcal{L}^{n} \varphi\right)(x)-\int \varphi(y) d \mu(y)
$$

for different values of $x$. For each given $x$ there is an associated renewal equation for $d_{n}$, from which functional equations of type (1) follow. Corollary 3.5 of the present paper is used in [2] to estimate a lower bound for $d_{n}$. The final result in [2] is that the correlation $c_{n}$ is of order $n^{-\gamma}, \gamma>1$.

\section{Kaluza's Theorem}

In this section, for the sake of completeness, we state and transcribe the proof of a result of T. Kaluza [3] that is used in the proof of our main theorem.

Theorem 2.1 ([3], Theorem 3). If

$$
\left(1+\sum_{n=1}^{\infty} \beta_{n} x^{n}\right)\left(1-\sum_{n=1}^{\infty} \alpha_{n} x^{n}\right)=1
$$

for two power series with positive radius of convergence, $\beta_{1}>0$, and

$$
\left|\begin{array}{cc}
\beta_{n-1} & \beta_{n} \\
\beta_{n} & \beta_{n+1}
\end{array}\right| \geq 0, \text { for all } n \geq 1
$$

(take $\beta_{0}=1$ ), then $\alpha_{n} \geq 0$, for all $n \geq 1$.

Remark. Condition (3) can be rephrased as a condition relating the geometric means $\left(\beta_{n+1}\right)^{2} \leq \beta_{n} \beta_{n+2}$ or still by saying that the sequence $\left(\beta_{n+1} / \beta_{n}\right)$ is nondecreasing. Note that (3) implies that $\beta_{n}>0, \forall n$. Indeed suppose we already know that $\beta_{n-1}>0$ and $\beta_{n}>0$. From (3) it follows that $\beta_{n-1} \beta_{n+1} \geq$ $\beta_{n}^{2}>0$, and then $\beta_{n+1}>0$. Since $1=\beta_{0}>0$ and $\beta_{1}>0$, the conclusion follows. Therefore (3) is equivalent to

$$
\left|\begin{array}{cc}
\beta_{m} & \beta_{n} \\
\beta_{m+1} & \beta_{n+1}
\end{array}\right| \geq 0, \text { for all } n \geq m .
$$

Proof of Theorem 2.1. From (2) it follows

$1+\left(\beta_{1}-\alpha_{1}\right) x+\left(\beta_{2}-\alpha_{1} \beta_{1}-\alpha_{2}\right) x^{2}+\left(\beta_{3}-\alpha_{1} \beta_{2}-\alpha_{2} \beta_{1}-\alpha_{3}\right) x^{3}+\cdots=1$, and, from this, the recursive relation

$$
\beta_{n}=\sum_{k=1}^{n} \alpha_{k} \beta_{n-k}
$$


where $\beta_{0}=1$. Solving (5) for $\alpha_{n}$, we get

$$
\alpha_{n+1}=\beta_{n+1}-\sum_{k=1}^{n} \alpha_{k} \beta_{n-k+1} .
$$

Multiplying (5) by $\beta_{n+1}$ and (6) by $\beta_{n}$, and adding the results together, it follows that

$$
\beta_{n} \alpha_{n+1}=\sum_{k=1}^{n}\left|\begin{array}{cc}
\beta_{n-k} & \beta_{n} \\
\beta_{n-k+1} & \beta_{n+1}
\end{array}\right| \alpha_{k}
$$

Note that from (4) it follows that all the determinants appearing in (7) are nonnegative. Combining this with the fact that $\beta_{n}>0$, for all $n$, and $\alpha_{0}>0$, we obtain, by induction on $n, \alpha_{n} \geq 0$, for all $n$.

\section{Main Theorem}

Given a series $\sum_{n=1}^{\infty} a_{n}$ of nonnegative terms satisfying

$$
\sum_{n=1}^{\infty} a_{n}=1
$$

it follows that the series

$$
f(x)=\sum_{n=1}^{\infty} a_{n} x^{n}
$$

has radius of convergence at least 1 , and satisfies $|f(x)|<1$ if $|x|<1$ and $f(1)=1$. Define

$$
h(x)=\frac{1}{1-f(x)}=\sum_{n=0}^{\infty} p_{n} x^{n} .
$$

Since also $h(x)=\frac{1}{1-f(x)}=\sum_{n=0}^{\infty}[f(x)]^{n}$, the series of $h(x)$ has radius of convergence 1 , and $p_{0}=1, p_{n}>0, \forall n$, and $\sum_{n=0}^{\infty} p_{n}=\infty$.

Theorem 3.1. Let $\sum_{n=1}^{\infty} a_{n}$ be a series of positive decreasing terms satisfying (8) and let $f(x)$ and $h(x)$ be defined by (9) and (10), respectively. If

$$
a_{2} \leq\left(1-a_{1}\right) a_{1}
$$

and

$$
r_{n+1}\left(a_{n}-a_{n+1}\right)-\left(a_{n+1}\right)^{2} \geq 0, \quad \forall n \geq 2,
$$

where $r_{n}=\sum_{m=n+1}^{\infty} a_{m}=1-\left(a_{1}+a_{2}+\cdots+a_{n}\right)$ is the tail of the series, then the sequence $\left(p_{n}\right)$ of coefficients of $h(x)$ is nonincreasing. 
Proof. Note that

$$
\begin{aligned}
h(x) & =\frac{1}{1-\sum_{n=1}^{\infty} a_{n} x^{n}}=\frac{1}{\sum_{n=1}^{\infty} a_{n}-\sum_{n=1}^{\infty} a_{n} x^{n}} \\
& =\frac{1}{(1-x) \sum_{n=1}^{\infty}\left(1+x+\cdots+x^{n-1}\right) a_{n}} \\
& =\frac{1}{1-x} \frac{1}{1+r_{1} x+r_{2} x^{2}+\cdots} .
\end{aligned}
$$

Let $\alpha_{n}$ be given by

$$
\frac{1}{1+r_{1} x+r_{2} x^{2}+\cdots}=1-\sum_{n=1}^{\infty} \alpha_{n} x^{n} .
$$

We then have

$$
h(x)=\left(\sum_{n=0}^{\infty} x^{n}\right)\left(1-\sum_{n=1}^{\infty} \alpha_{n} x^{n}\right)
$$

and, hence, $p_{n}=1-\alpha_{1}-\alpha_{2}-\cdots-\alpha_{n}$. Therefore to show that $\left(p_{n}\right)$ is nonincreasing, it suffices to show that $\alpha_{n} \geq 0$, for all $n$. Since $r_{1}=a_{1}>0$, by Theorem 2.1, it suffices to verify that

$$
\left(r_{1}\right)^{2} \leq r_{2}
$$

and that

$$
\left(r_{n}\right)^{2} \leq r_{n-1} r_{n+1}, \quad \forall n \geq 2 .
$$

But (13) is equivalent to (11), and (14) follows from (12).

Corollary 3.2. Let $\sum_{n=1}^{\infty} a_{n}$ be a series of positive decreasing terms satisfying (8) and let $f(x)$ and $h(x)$ be defined by (9) and (10), respectively. If the sequence $\left(a_{n}\right)$ satisfies (11) and

$$
\left(a_{n+1}\right)^{2} \leq a_{n} a_{n+2},
$$

for all $n \geq 2$, then the sequence $\left(p_{n}\right)$ of coefficients of $h(x)$ is nonincreasing.

Proof. It suffices to show that

$$
\frac{\left(a_{n+1}\right)^{2}}{a_{n}-a_{n+1}} \leq a_{n+2}+a_{n+3}+a_{n+4}+\cdots .
$$


But

$$
\frac{\left(a_{n+1}\right)^{2}}{a_{n}-a_{n+1}}=a_{n+1}\left[\frac{a_{n+1}}{a_{n}}+\left(\frac{a_{n+1}}{a_{n}}\right)^{2}+\left(\frac{a_{n+1}}{a_{n}}\right)^{3}+\cdots\right] .
$$

Therefore for (16) it is enough to have each term on the right-hand side of (16) less than or equal to the corresponding term on the right-hand side of (17); i.e., it is sufficient that

$$
\left(a_{n+1}\right)^{k} \leq\left(a_{n}\right)^{k-1} a_{n+k}, \forall k \geq 2 \text {, and } \forall n \geq 1 .
$$

But (15) is a special case of (18), and the general case follows by an induction argument.

Lemma 3.3. If $\sum_{n=1}^{\infty} a_{n}=1$ is a series of positive terms satisfying (15) for all $n \geq 1$, then (11) holds.

Proof. Since $\left(a_{n+1} / a_{n}\right)$ is nondecreasing, $\left(\frac{a_{2}}{a_{1}}\right)^{n} \leq \frac{a_{2}}{a_{1}} \frac{a_{3}}{a_{2}} \cdots \frac{a_{n+1}}{a_{n}}$, for all $n \geq 1$. Therefore $a_{n+1} \geq \frac{\left(a_{2}\right)^{n}}{\left(a_{1}\right)^{n-1}}$, for all $n \geq 1$. But combining this with $1=a_{1}+a_{2}+a_{3}+\cdots$ yields $a_{2} \leq\left(1-a_{1}\right) a_{1}$.

Corollary 3.4. Let $\sum_{n=1}^{\infty} a_{n}$ be a series of positive decreasing terms, and let $f(x)$ and $h(x)$ be defined by (9) and (10), respectively. If the sequence $\left(a_{n}\right)$ satisfies (15) for all $n \geq 1$, then the sequence $\left(p_{n}\right)$ of coefficients of $h(x)$ is nonincreasing.

Remark. The hypotheses of Corollary 3.4 are slightly weaker than those of Corollary 3.2. In practice, what makes the former easier to apply than the latter, is that for a sequence to satisfy a condition like $\left(a_{n+1}\right)^{2} \leq a_{n} a_{n+2}$, it suffices that some positive multiple of the sequence satisfies it. The same is not true for the condition $a_{2} \leq\left(1-a_{1}\right) a_{1}$. Nevertheless a series may satisfy the hypotheses of Corollary 3.2 but not those of Corollary 3.4. An example is

$$
f(x)=\frac{1}{2} x+\frac{1}{4} x^{2}+\frac{1}{16} x^{3}+\frac{1}{16} \frac{3}{4} x^{4}+\frac{1}{16}\left(\frac{3}{4}\right)^{2} x^{5}+\cdots .
$$

Corollary 3.5. Under the same general assumptions as above, if $\left(a_{n}\right)$ satisfies

$$
a_{n}=F(n), \text { for all } n \geq 1,
$$

with $F:[1, \infty) \longrightarrow(0, \infty)$ a log-convex function (i.e., a function such that $\log (F(x))$ is convex), then the same conclusion of Corollary 3.4 holds; i.e., the Taylor coefficients of $h(x)$ form a nonincreasing sequence. 


\section{Application}

For any real $\gamma>1$, let $f_{\gamma}(x)=\sum_{n=1}^{\infty} \frac{n^{-\gamma}}{\zeta(\gamma)} x^{n}$, where $\zeta(x)=\sum_{n=1}^{\infty} \frac{1}{n^{x}}$ is the Riemann Zeta function. Here, $a_{n}=F(n)$, with $F(x)=\frac{x^{-a}}{\zeta(a)}$, and

$$
\log (F(x))=-\log (\zeta(a))-a \log (x)
$$

is a convex function. By Corollary 3.5, the Taylor coefficients of $h(x)=\frac{1}{1-f(x)}$ are nonincreasing.

\section{Acknowledgement}

The author would like express his gratitude to Prof. A. Lopes for posing the question that is the object of the present investigation, for the encouragement, and for invaluable suggestions. He also thanks the Department of Mathematical Sciences of the University of Delaware for the support.

\section{References}

[1] N. K. Bary, A Treatise on Trigonometric Series, vol II, Pergamon Press, 1964.

[2] A. Fisher and A. Lopes, Exact bounds for the polynomial decay of correlation, $1 / f$ noise and the CLT for the equilibrium state of a non-Hölder potential, it Nonlinearity, 14 (2001), 1071-1104.

[3] T. Kaluza, Über die Koeffizienten reziproker Potenzreihen, Math. Zeit., 28 (1928), 161-170.

[4] P. Manneville, Intermittency, self-similarity and $1 / f$ spectrum in dissipative dynamical systems, J. Physique, 41 (1980), 1235-1243.

[5] A. Postnikov, Tauberian Theory and its applications, Proc. Steklov Inst. Math, AMS, 1980. 